\title{
Amyloid-beta related angiitis of the central nervous system: case report and topic review
}

\author{
Amre Nouh ${ }^{1}$, Ewa Borys ${ }^{2}$, Angelica K. Gierut ${ }^{3}$ and José Biller ${ }^{1 *}$ \\ ${ }^{1}$ Department of Neurology, Stritch School of Medicine, Loyola University Chicago, Maywood, IL, USA \\ 2 Section of Neuropathology, Department of Pathology, Stritch School of Medicine, Loyola University Chicago, Maywood, IL, USA \\ ${ }^{3}$ Section of Allergy, Immunology and Rheumatology, Stritch School of Medicine, Loyola University Chicago, Maywood, IL, USA
}

\section{Edited by:}

Osvaldo Fustinoni, Universidad de

Buenos Aires, Argentina

\section{Reviewed by:}

Maria Zurru, Hospital Italiano de

Buenos Aires, Argentina

Emilia Gatto, Sanatorio de la Trinidad

Mitre, Argentina

\section{${ }^{*}$ Correspondence:}

José Biller, Department of Neurology,

Loyola University Chicago, Stritch

School of Medicine, 2160 S, 1st

Avenue, Building 105, Room 2700

Maywood, IL 60153, USA

e-mail: jbiller@lumc.edu
Amyloid-beta related angiitis (ABRA) of the central nervous system (CNS) is a rare disorder with overlapping features of primary angiitis of the CNS and cerebral amyloid angiopathy. We evaluated a 74-year-old man with intermittent left sided weakness and MRI findings of leptomeningeal enhancement, vasogenic edema, and subcortical white matter disease proven to have ABRA. We discuss clinicopathological features and review the topic of ABRA.

Keywords: amyloid-beta related angiitis, primary angiitis of the central nervous system, cerebral amyloid angiopathy, leptomeningeal enhancement, cerebral amyloid angiopathy-related inflammation

\section{CASE REPORT}

A 74-year-old man with a past medical history of chronic lymphocytic leukemia (CLL) stage 0 (not receiving treatment), renal cell carcinoma (clear cell type, nuclear grade II-untreated), hypertension, and hyperlipidemia awoke one morning with numbness of the thumb and first two fingers of the left hand "marching" up to the left arm over the course of few seconds. This was immediately followed by drooling from the left corner of his mouth. The entire episode lasted $<15 \mathrm{~s}$ and was followed by complete resolution. The following day, he had an episode of slurred speech and left facial weakness, which prompted evaluation at a local hospital where his symptoms again completely resolved. He was admitted for further investigation. Contrast-enhanced brain MRI showed leptomeningeal enhancement and increased signal intensity on T2 fluid attenuated inversion recovery (FLAIR) sequences on the right temporal lobe, as well as moderate subcortical and periventricular T2 signal abnormalities suggestive of white matter disease (Figures 1A-D). He was diagnosed as possibly having "herpes encephalitis" and intravenous Acyclovir was administered. Cerebrospinal fluid (CSF) analysis showed a white blood cell (WBC) count of 2 cells $/ \mu \mathrm{L}$, red blood cell count of 83 cells $/ \mu \mathrm{L}$, glucose of $61 \mathrm{mg} / \mathrm{dL}$, and protein content of $282 \mathrm{mg} / \mathrm{dL}$ (normal range $15-45 \mathrm{mg} / \mathrm{dL}$ ). Herpes simplex virus PCR was negative, and thus, Acyclovir was discontinued. Other laboratory analyses included an erythrocyte sedimentation rate (ESR) of $40 \mathrm{~mm} / \mathrm{h}$, a positive antinuclear antibody (titer unavailable), and negative anti-neutrophil cytoplasmic antibodies (ANCAs). During that hospital stay, he had several similar episodes of marching numbness involving his left hand and facial asymmetry without loss of consciousness or adventitious limb movements. Electroencephalogram (EEG) was normal. He received Levetiracetam $500 \mathrm{mg}$ twice daily that was subsequently increased to $750 \mathrm{mg}$ twice daily. He was discharged home without a definite diagnosis. However, the next day, he developed severe pain over the right temple and presented to another institution where a second MRI showed similar findings, and a right temporal leptomeningeal biopsy showed intramural as well as perivascular inflammatory infiltrates with granulomatous features in the leptomeninges and penetrating blood vessels associated with vasculopathic changes, as well as positive labeling for beta-amyloid on immunohistochemical studies consistent with amyloid-beta related angiitis (ABRA). He was then referred to our Medical Center for follow-up and management. Upon initial evaluation by us, neurologic examination was normal. However, 2 days later, he had mild right lower extremity weakness and slurred speech prompting hospital admission to our institution. A third brain MRI showed a new left frontal leptomeningeal area of enhancement and scattered FLAIR abnormalities on the left temporal lobe without evidence of acute infarction. MRI of the cervical spine showed no intrinsic cord changes (Figures 2A-D). EEG showed diffuse slow wave abnormalities. He received a 5-day course of $1000 \mathrm{mg}$ intravenous methylprednisolone followed by a daily maintenance dose of $60 \mathrm{mg}$ prednisone. Mycophenolate mofetil was chosen for immunosuppression in lieu of cyclophosphamide, given his CLL. The patient has remained neurologically stable since hospital discharge.

\section{DISCUSSION}

Amyloid-beta related angiitis is a predominantly granulomatous angio-destructive inflammatory mediated disease affecting leptomeningeal and cortical vessels characterized by meningeal lymphocytosis and abundant amyloid-beta deposition within the vessel walls $(1,2)$. Although sharing characteristics of both PACNS and cerebral amyloid angiopathy (CAA), ABRA has some differentiating clinical and pathological features. PACNS has an annual 


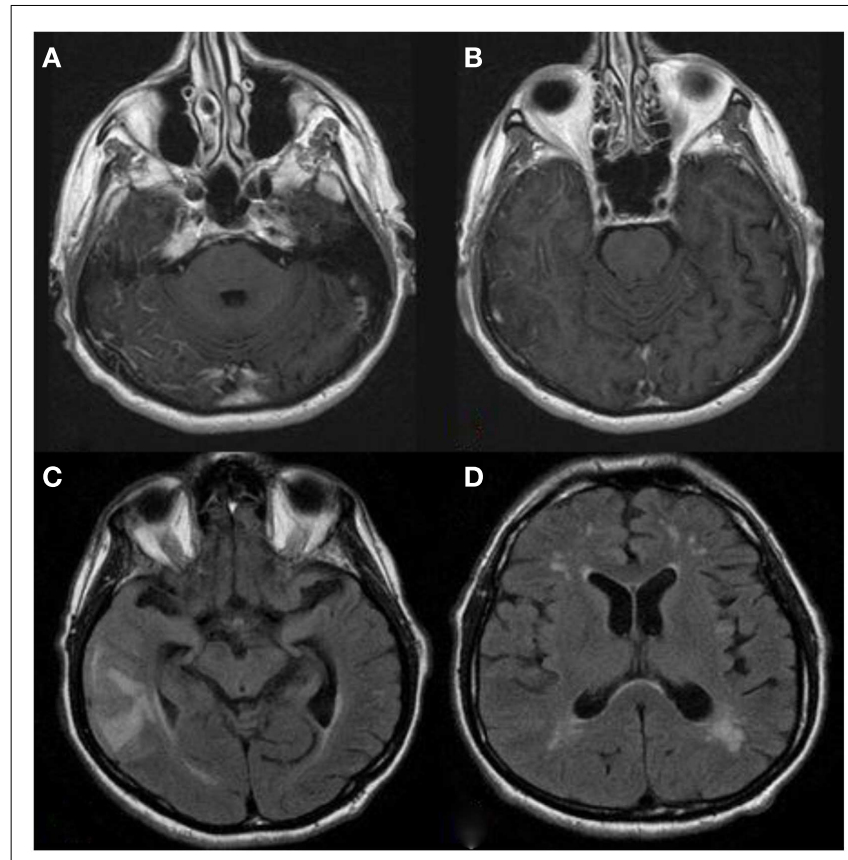

FIGURE 1 | (A,B) T1 contrast-enhanced axial MRI shows evidence of right temporal leptomeningeal enhancement. (C) T2 FLAIR shows right temporal lobe vasogenic edema. (D) Scattered subcortical white matter disease.

incidence of 2.4:1,000,000 and is diagnosed by the presence of acquired neurologic or psychiatric deficits and classic angiographic or histologic findings of CNS angiitis, unaccounted for by other systemic causes or disease mimics (3). CAA, characterized by betaamyloid deposition (primarily the more soluble form of A $\beta$ 1-40) within leptomeningeal and cortical vessels is found in 30-50\% of asymptomatic elderly subjects and in approximately $80 \%$ of patients with Alzheimer's disease. Moreover, CAA accounts for one third of all lobar hemorrhages in elderly subjects (4). A neuropathological variant of ABRA characterized by non-destructive perivascular inflammatory infiltration and amyloid deposition is known as CAA-related inflammation (CAA-RI) $(5,6)$. Patients with CAA-RI share most of the clinical features of ABRA, and most often present with seizures, and/or cognitive decline $(5,6)$. Both entities share similar clinical features that respond similarly to treatment (2).

The pathophysiology of ABRA is not fully understood. Whether amyloid-beta deposits initiate a vasculitic response or rather angiopathic-mediated inflammation leads to beta-amyloid deposition remains debatable. One theory suggests an immunologic response toward amyloid-beta, resulting in leptomeningeal and parenchymal inflammation with increased amyloid-beta deposition and clearance of amyloid-beta in the parenchyma (1). Decreasing levels of $A \beta \quad 1-40$ and $A \beta$ 1-42 CSF autoantibodies in two patients (one CAA-RI one ABRA) after steroid therapy further suggest an autoimmune response (2). The presence of partially activated CD4+ T-cells in the CSF (7), vasculitic changes in response to vascular amyloid in transgenic mice that develop CAA (8), and favorable response to steroids and immunosuppressive therapy supports an immune-mediated pathogenesis.

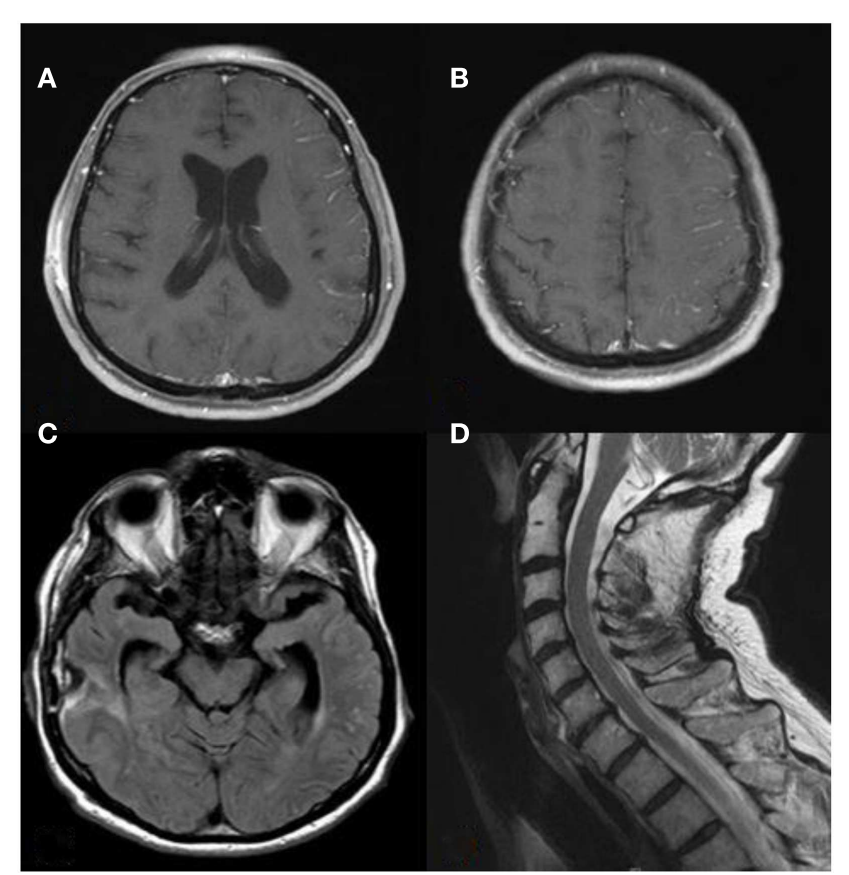

FIGURE 2 | (A,B) T1 contrast-enhanced axial MRI shows new left frontal leptomeningeal enhancement. (C) T2 FLAIR shows new left temporal lobe changes. (D) T2 sagittal view of the cervical spine showed no evidence of intrinsic cord abnormalities.

Amyloid-beta related angiitis usually presents in the seventh decade of life (mean age of 67 years) - without gender predilection (1). The main clinical features of ABRA, in order of frequency, include mental status and cognitive changes followed by headaches, seizures, and focal neurologic deficits $(1,2,9,10)$. In addition, patients may rarely present with uveomeningitis (11), posterior reversible encephalopathy-like manifestations, (10) or progression to coma (12).

An abnormal ESR has been reported in $12-38 \%$ of patients with $\operatorname{ABRA}(1,2)$. In the series by Scolding et al., none of the 21 patients tested had positive ANCAs. In contrast, CSF abnormalities are extremely common and present in $85 \%$ of patients. Protein content elevation has been found in $60-90 \%$ of patients, with a median level of $100 \mathrm{mg} / \mathrm{dL}$. Primarily, lymphocytic pleocytosis with a WBC count $>5$ cells has been observed in $50 \%$ of cases $(1,2)$. CSF oligoclonal bands have been absent in all patients tested by Scolding et al. (1). In one report, CSF tau and A $\beta 42$ were found to be normal in one patient with positive oligoclonal bands (13). CSF flow cytometric analyses have shown predominantly CD4+ T-cells (7). The most common APOE genotype found when compared to non-inflammatory CAA patients was APOE4, present in $71-76 \%$ of tested patients $(5,6)$.

The most common neuroimaging findings of ABRA are gadolinium-enhancing leptomeninges (2). Other findings include non-specific white matter changes, vasogenic edema, mass lesions, infarctions, and/or intracerebral hemorrhages (1, 2, 9, 10). Leptomeningeal or cortical hemosiderin deposits are observed in some cases $(5,7,14)$ but unlike CAA, cerebral hemorrhages are less 
frequent $(1,2)$. Angiographic abnormalities are also infrequent $(2,5)$. Most common EEG abnormalities are diffuse slow wave changes (1).

Treatment of ABRA and CAA-RI include corticosteroids and immunosuppression, primarily with cyclophosphamide $(1,2,5$, 6). In one series, $5 / 6$ patients with CAA-RI clinically improved with treatment, returning to their premorbid status. Four out of these five patients demonstrated MRI improvement, and only two deaths were reported between 2 and 3 years from initial presentation (5). In another series of 12 patients with CAA-RI, 7 improved within 1-2 weeks of treatment, 3 patients had relapses while not receiving treatment, and 2 patients showed no clinical response (6). In the series by Salvari et al., 10/28 patients with ABRA were treated with steroids alone, and $13 / 28$ patients were treated with a combination of steroids and immunosuppression for 5-8 months (nine cyclophosphamide, four other agents). Those treated with steroids + immunosuppression fared better than patients with CAA but not differently than patients with PACNS. In the series by Scolding et al., approximately one in four patients required a surgical intervention such as CSF diversion, mass lesion resection, or craniotomy. Mortality in ABRA has been reported to be between 7 and $44 \%(1,2)$.

Amyloid-beta related angiitis and CAA-RI are unusual neuropathological diagnoses. Mental status or cognitive changes, gadolinium-enhancing meninges, and elevated CSF protein content are frequent findings. In contrast to PACNS, patients with ABRA have better response to immunosuppressive therapy. Differentiating prognostic and management features between ABRA and CAA-RI require further refinement.

\section{REFERENCES}

1. Scolding NJ, Joseph F, Kirby PA, Mazanti I, Gray F, Mikol J, et al. A $\beta$-related angiitis: primary angiitis of the central nervous system associated with cerebral amyloid angiopathy. Brain (2005) 128(3):500-15.

2. Salvarani C, Hunder GG, Morris JM, Brown RD, Christianson T, Giannini C. A $\beta$-related angiitis comparison with CAA without inflammation and primary CNS vasculitis. Neurology (2013) 81(18):1596-603. doi:10.1212/WNL. 0b013e3182a9f545

3. Hajj-Ali RA, Singhal AB, Benseler S, Molloy E, Calabrese LH. Primary angiitis of the CNS. Lancet Neurol (2011) 10(6):561-72. doi:10.1016/S1474-4422(11) 70081-3

4. Biffi A, Greenberg SM. Cerebral amyloid angiopathy: a systematic review. J Clin Neurol (2011) 7(1):1-9. doi:10.3988/jen.2011.7.1.1
5. Eng JA, Frosch MP, Choi K, Rebeck GW, Greenberg SM. Clinical manifestations of cerebral amyloid angiopathy-related inflammation. Ann Neurol (2004) 55(2):250-6. doi:10.1002/ana.10810

6. Kinnecom C, Lev MH, Wendell L, Smith EE, Rosand J, Frosch MP, et al. Course of cerebral amyloid angiopathy-related inflammation. Neurology (2007) 68(17):1411-6. doi:10.1212/01.wnl.0000260066.98681.2e

7. Melzer N, Harder A, Gross CC, Wolfer J, Stummer W, Niederstadt T, et al. CD4+ T Cells predominate in cerebrospinal fluid and leptomeningeal and parenchymal infiltrates in cerebral amyloid $\beta$-related angiitis. Arch Neurol (2012). 69(6):773-7. doi:10.1001/archneurol.2011.2441

8. Winkler DT, Bondolfi L, Herzig MC, Jann L, Calhoun ME, Wiederhold KH, et al. Spontaneous hemorrhagic stroke in a mouse model of cerebral amyloid angiopathy. J Neurosci (2001) 21(5):1619-27.

9. Salvarani C, Brown RD, Calamia KT, Christianson TJH, Huston J, Meschia JF, et al. Primary central nervous system vasculitis: comparison of patients with and without cerebral amyloid angiopathy. Rheumatology (2008) 47(11):1671-7. doi:10.1093/rheumatology/ken328

10. Rigby H, Easton A, Bhan V. Amyloid $\beta$-related angiitis of the central nervous system: report of 3 cases. Can J Neurol Sci (2011) 38(4):626-30.

11. Child ND, Braksick SA, Flanagan EP, Keegan BM, Giannini C, Kantarci OH. Amyloid- $\beta$-related angiitis presenting as a uveomeningeal syndrome. Neurology (2013) 81(20):1796-8. doi:10.1212/01.wnl.0000435560.00234.a7

12. Lee K. Amyloid $\beta$-related angiitis causing coma responsive to immunosuppression. Case Rep Pathol (2012) 2012:3.

13. Kurian M, Burkhardt K, Assal F, Kövari E, Horvath J. Amyloid plaques and intraneuronal tau inclusions in A-beta-related angiitis (ABRA). Neuropathol Appl Neurobiol (2012) 38(4):391-4. doi:10.1111/j.1365-2990.2011.01245.x

14. Tschampa HJ, Niehusmann P, Marek M, Mueller CA, Kuchelmeister K, Urbach H. MRI in amyloid $\beta$-related brain angiitis. Neurology (2009) 73(3):247-247. doi:10.1212/WNL.0b013e3181ae7cd0

Conflict of Interest Statement: The authors declare that the research was conducted in the absence of any commercial or financial relationships that could be construed as a potential conflict of interest.

Received: 31 December 2013; accepted: 17 January 2014; published online: 04 February 2014.

Citation: Nouh A, Borys E, Gierut AK and Biller J (2014) Amyloid-beta related angiitis of the central nervous system: case report and topic review. Front. Neurol. 5:13. doi: 10.3389/fneur.2014.00013

This article was submitted to Neurology Education, a section of the journal Frontiers in Neurology.

Copyright (c) 2014 Nouh, Borys, Gierut and Biller. This is an open-access article distributed under the terms of the Creative Commons Attribution License (CC BY). The use, distribution or reproduction in other forums is permitted, provided the original author(s) or licensor are credited and that the original publication in this journal is cited, in accordance with accepted academic practice. No use, distribution or reproduction is permitted which does not comply with these terms. 


\section{APPENDIX}

\section{PATHOLOGY}

Brain biopsy showed cortical and subcortical white matter with perivascular chronic intramural inflammatory infiltrates with fibrinoid deposition along with granulomatous features involving meningeal and superficial penetrating arteries. In many instances, the inflammatory changes were associated with deposition of amorphous eosinophilic material in the vessel walls consistent with amyloid. Congo red and $\beta$-amyloid immunohistochemical stain were positive.

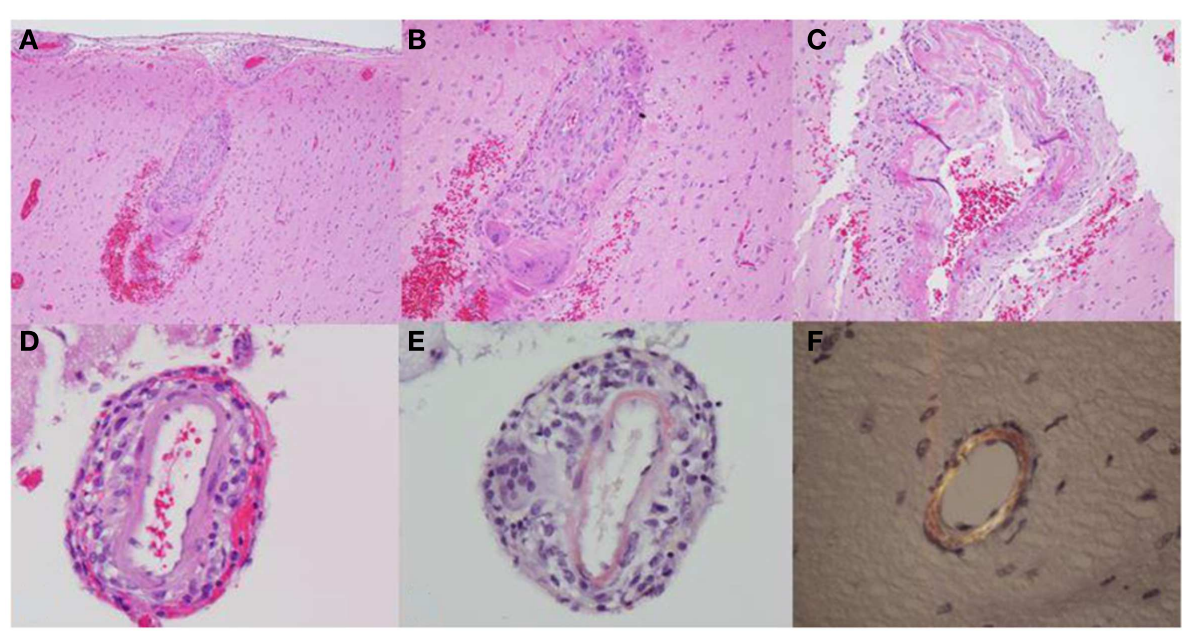

FIGURE A1 | (A,B) Leptomeningeal and cortical blood vessels with granulomatous inflammation and surrounding hemorrhage, $40 \times$ and $100 \times$. (C) Cortical vessel with inflammation and fibrinoid necrosis, 200×. (D) Vessel with granulomatous inflammation and amyloid deposition, 400×. (E) Congo red stain positive for amyloid, $400 \times$. (F) Congo stain showing characteristic apple-green birefringence under polarized light, $400 \times$. 